\title{
Interaction region indicator (IRI): A very simple real space function clearly revealing both chemical bonds and weak interactions
}

\author{
Tian $\mathrm{Lu},{ }^{[\mathrm{a}], *}$ Qinxue Chen ${ }^{[\mathrm{a}]}$
}

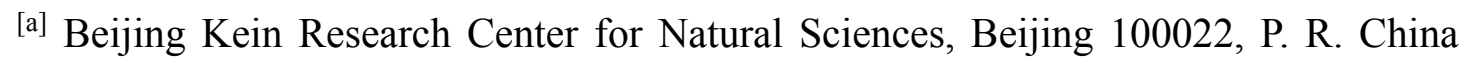
(http://www.keinsci.com)

* Correspondence author. E-mail: sobereva@sina.com

\author{
Author information \\ Tian Lu ORCiD: 0000-0002-1822-1229 E-mail: sobereva@sina.com \\ Qinxue Chen: ORCiD: 0000-0003-0155-2387 E-mail: qinxue_chen@sina.com
}

\begin{abstract}
Graphically revealing interaction regions in a chemical system enables chemists to notice the areas at a glance where significant interactions have formed, it is very helpful in studying chemical bonds, intermolecular and intramolecular interactions. Reduced density gradient (RDG) has already been widely employed in literatures to visually exhibit weak interaction regions, in fact it also has the ability of revealing chemical bonding regions. Unfortunately, RDG cannot clearly show both types of the interactions at the same time. In this paper, we propose a new real space function named interaction region indicator (IRI), which is a slight modification on RDG. We found IRI can reveal chemical bonding and weak interaction regions equally well, this brings great convenience in the study of various chemical systems as well as chemical reactions. It is noteworthy that IRI has simpler definition, lower computational cost and better graphical effect than the density overlap regions indicator (DORI), which has similar purpose to IRI. In this article IRI is also compared with atom-in-molecules (AIM) topology analysis of electron density, we demonstrated that IRI has the ability to reveal additional interactions to provide chemists a more complete picture. In addition, we put forward a variant of IRI named IRI- $\pi$, which is dedicated to reveal interactions of $\pi$
\end{abstract}


electrons. It is found that IRI- $\pi$ can not only distinguish type of $\pi$ interactions but can also exhibit $\pi$-interaction strength. IRI and IRI- $\pi$ have been efficiently implemented in our freely available Multiwfn wavefunction analysis code, it is expected that they will become new useful members of computational chemists' toolbox in studying chemical problems.

Keywords: electron density; reduced density gradient; hydrogen bond; van der Waals interaction; chemical bond; atom-in-molecules; Multiwfn 


\section{Introduction}

Interatomic interactions are ubiquitous in chemical systems. If all regions involving notable interatomic interactions can be simultaneously graphically exhibited as a picture, undoubtedly it will bring great convenience for chemists to capture characteristics of the current system at a glance, and it will be very helpful for studying chemical bonds, analyzing intermolecular and intramolecular interactions.

Reduced density gradient (RDG) is a dimensionless form of gradient of electron density, it is expressed as

$$
\operatorname{RDG}(\mathbf{r})=\frac{1}{2\left(3 \pi^{2}\right)^{1 / 3}} \frac{|\nabla \rho(\mathbf{r})|}{[\rho(\mathbf{r})]^{4 / 3}}
$$

where $\rho$ is electron density and $\mathbf{r}$ is coordinate vector. In the so-called noncovalent interaction (NCI) method proposed by Yang et al., ${ }^{[1]}$ isosurface map of RDG at low electron density area has been employed for visually revealing regions related to various kinds of noncovalent interactions as well as the regions showing notable steric effect. In fact, by properly choosing the isovalue, isosurface map of RDG can also clearly reveal covalent bond regions, though this point has been largely neglected. As will be shown later, it is difficult to simultaneously reveal both noncovalent and covalent interactions in a single RDG map, because the optimal isovalues for showing the two kinds of interactions are markedly different. We note that in order to exhibit both covalent and noncovalent interactions at the same time, some researchers employed RDG and electron localization function (ELF) in combination, as ELF has a strong capacity of revealing covalent interactions. ${ }^{[2-5]}$ However, simultaneously considering two functions in an analysis is evidently very inconvenient.

Density overlap regions indicator (DORI) aims at revealing covalent and noncovalent interactions in an equal footing, ${ }^{[6]}$ it is expressed as

$$
\operatorname{DORI}(\mathbf{r})=\frac{\theta(\mathbf{r})}{1+\theta(\mathbf{r})}
$$

where 


$$
\theta(\mathbf{r})=\frac{\left\{\nabla\left[\frac{\nabla \rho(\mathbf{r})}{\rho(\mathbf{r})}\right]^{2}\right\}^{2}}{\left[\frac{\nabla \rho(\mathbf{r})}{\rho(\mathbf{r})}\right]^{6}}
$$

The value range of DORI is $[0,1]$. In our view, there are two shortcomings of DORI: (1) The definition of DORI is somewhat too complicated, and its calculation requires second order derivative of electron density, which is more difficult to implement and evaluate than RDG (2) Graphical effect of DORI isosurface is often unsatisfactory, and DORI is numerically noise in some regions.

In this work we propose a new real space function named interaction region indicator (IRI) by slightly modifying RDG. The purpose of IRI is similar to DORI, however IRI has a much simpler definition and more satisfactory graphical effect. In addition, the computational cost of IRI is lower than that of DORI, since IRI only involves electron density and its gradient. IRI has been supported by our freely available wavefunction analysis code Multiwfn, ${ }^{[7]}$ the use is quite easy and the calculation is fast. Multiwfn can be freely obtained at http://sobereva.com/multiwfn.

In the rest of this article, we will first introduce the definition of IRI and then compare IRI analysis with other methods. Next, the value of IRI will be demonstrated through a series of examples. After that, we will discuss a variant of IRI named IRI- $\pi$, which is useful in characterizing $\pi$-interactions. Finally we conclude the whole article. For all examples presented in this article, calculations of grid data were realized by Multiwfn version 3.8(dev), then isosurface maps were rendered by VMD 1.9.3 software. ${ }^{[8]}$ Geometry optimization and generation of electronic wavefunction were finished by Gaussian 16 A.03. ${ }^{[9]}$ Unless otherwise specified, B3LYP-D3(BJ) exchangecorrelation functional $^{[10,11]}$ in combination with SDD pseudopotential basis set ${ }^{[12]}$ for transition metals and $6-311 \mathrm{G}^{* *}$ basis $\mathrm{set}^{[13]}$ for other elements were employed for all calculations. Atomic unit is adopted for all quantities mentioned in the later sections. 


\section{Theory}

\subsection{Definition of IRI}

IRI is simply defined as follows

$$
\operatorname{IRI}(\mathbf{r})=\frac{|\nabla \rho(\mathbf{r})|}{[\rho(\mathbf{r})]^{a}}
$$

where $a$ is an adjustable parameter, $a=1.1$ is adopted for standard definition of IRI. IRI is essentially the gradient norm of electron density weighted by scaled electron density. Note that if $a=4 / 3$, IRI only differs from RDG by a constant prefactor. Obviously, IRI can be immediately implemented in any code that already supported RDG. As will be shown, by properly choosing isovalue, isosurfaces of IRI are able to exhibit various kinds of interaction regions.

Analogous to NCI analysis, ${ }^{[1]} \operatorname{sign}\left(\lambda_{2}\right) \rho$ function can be mapped on IRI isosurfaces by different colors to vividly show nature of the interaction regions revealed by IRI. The $\operatorname{sign}\left(\lambda_{2}\right)$ denotes the sign of the second largest eigenvalue of Hessian of $\rho$, which has certain ability to distinguish attractive and repulsive interactions, see Refs. [1] and [7] for details. The region showing relatively high $\rho$ and thus large magnitude of $\operatorname{sign}\left(\lambda_{2}\right) \rho$ implies a relatively strong interaction, while the area with low $\rho$ and thus small $\operatorname{sign}\left(\lambda_{2}\right) \rho$ does not participate in a noticeable interaction, or the interaction can be at most attributed to interatomic vdW interaction, which is known to be fairly weak. Our recommended coloring method of $\operatorname{sign}\left(\lambda_{2}\right) \rho$ on IRI isosurfaces is shown in Fig. 1, the corresponding chemical explanations are also given. From the mapped colors of $\operatorname{sign}\left(\lambda_{2}\right) \rho$ one can easily identify nature of interactions revealed by IRI isosurfaces, this coloring method will be employed for all figures given later. 


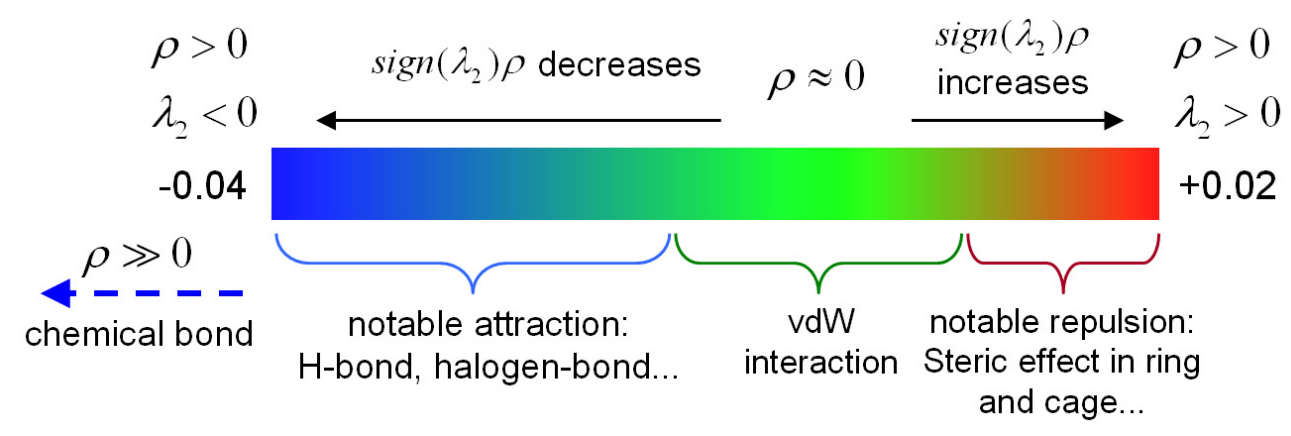

Fig. 1 Standard coloring method and chemical explanation of $\operatorname{sign}\left(\lambda_{2}\right) \rho$ on IRI isosurfaces.

\subsection{Comparison between IRI and RDG analyses}

In order to show what is the advantage of IRI compared to RDG in reveal interactions and demonstrate the importance of our modification on RDG, let us take phenol dimer as an example, which contains covalent bonds, an H-bond and vdW interactions. Fig. 2 shows RDG isosurface maps at different isovalues. It is easy to recognize that RDG is incapable of simultaneously revealing both covalent and noncovalent interactions in a single map. Isosurfaces of $\mathrm{RDG}=0.5$ display vdW, $\mathrm{H}$ bond and steric interactions clearly, however various chemical bonds cannot be distinguished. In the case of $\mathrm{RDG}=0.25$, covalent bond regions are nicely revealed by the isosurfaces, unfortunately, the noncovalent interaction regions can hardly be visualized. In contrast, the isosurfaces of IRI $=1.1$ in Fig. 2 equally well reveal both covalent and noncovalent interaction regions, showing the unique value of IRI. The parameter $a$ in IRI plays a key role in balancing the representation of covalent and noncovalent interactions, the $a=1.1$ was empirically determined by us through searching for the best exhibition of all kinds of interactions in multiple chemical systems. Note that there is no strict criterion for the selection of IRI isovalue, according to our experiences IRI $=1.0$ works well for almost all systems and thus it is chosen as the default value in the IRI plotting script provided by Multiwfn program. However, one can also try to slightly alter the isovalue to gain a better graphical effect for the system under study. 


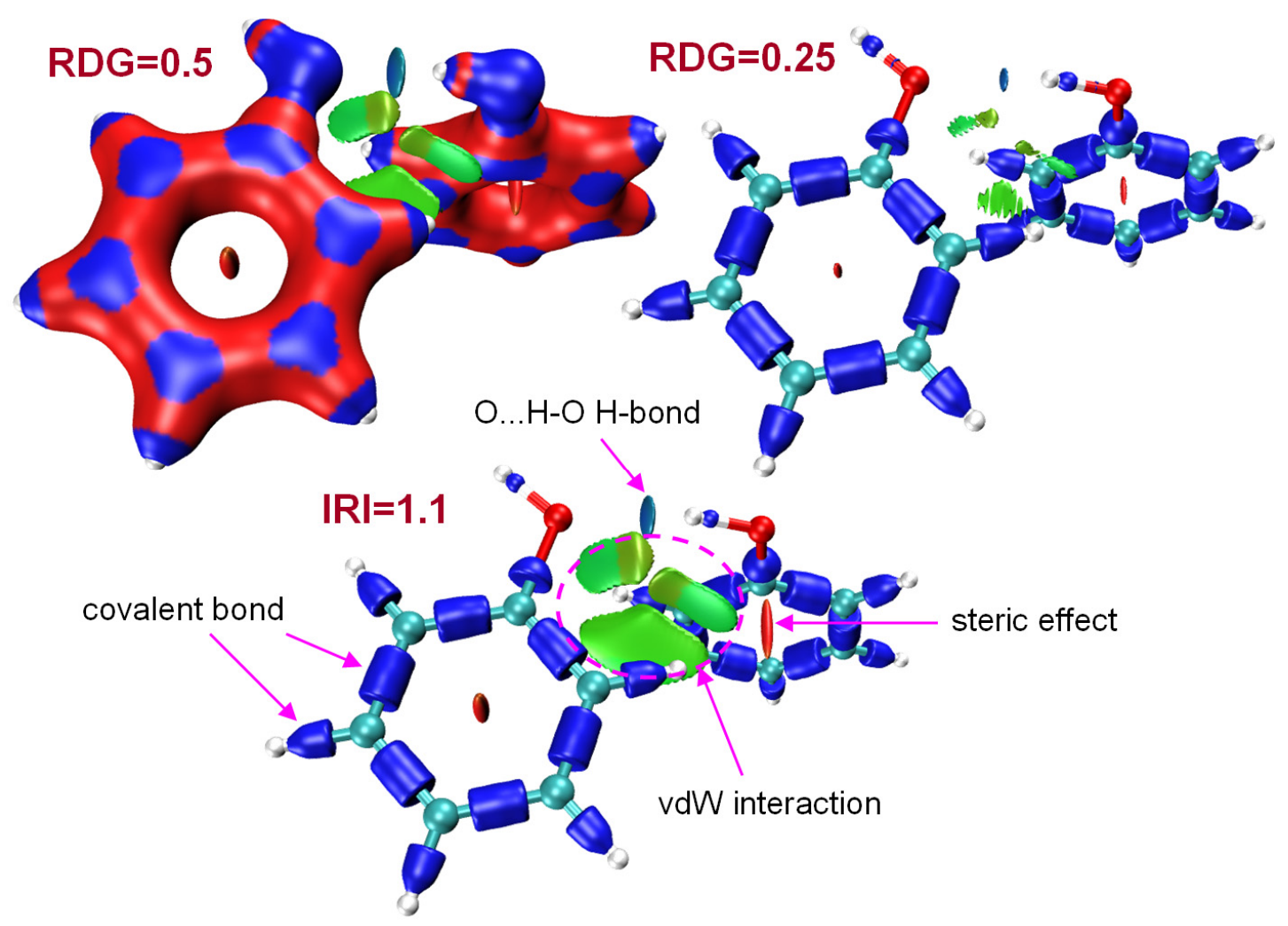

Fig. 2 Isosurface map of $\mathrm{RDG}=0.5, \mathrm{RDG}=0.25$ and IRI $=1.1 . \operatorname{sign}\left(\lambda_{2}\right) \rho$ is mapped on the isosurfaces according to coloring method of Fig. 1. Some featured regions in IRI map are labelled.

\subsection{Comparison between IRI and DORI analyses}

In this section we compare graphical effect of IRI and DORI via two examples to demonstrate the unique advantage of IRI compared to DORI.

Experimental synthesis and theoretical characterization of diamantanediamantane (7-7) have been reported in Ref. [14]. 7-7 can be thought of as two diamantane molecules covalently bonded. Because of the large size of diamantane, the $\mathrm{C}-\mathrm{C}$ bond linking the two diamantanes is evidently longer than a typical $\mathrm{C}-\mathrm{C}$ single bond due to steric effect between the two diamantane moieties. IRI $=1.0$ and DORI $=$ 0.96 isosurface maps of 7-7 are given in Fig. 3. Note that the shape of DORI isosurfaces is quite sensitive to the choice of isovalue, so the DORI map corresponding to isovalues 
of 0.92, 0.94 and 0.98 are also provided in Fig. S1. From Fig. 3 it can be seen that IRI nicely revealed all kinds of interactions in 7-7, including all covalent bonds, steric effect within each diamantane unit, as well as the steric repulsion and vdW interaction between the two diamantane units. Although the DORI map conveys similar information, its graphical effect is much poorer. It can be seen that there are many noisy or fragmentary isosurfaces between hydrogen atoms in each diamantane, and the broad isosurface exhibiting interaction between the two diamantanes has a ugly and rough edge. These visual issues are not caused by choice of grid quality because the current grid spacing is already very small (0.12 Bohr), but due to the inherent shortcoming of the definition of DORI. In addition, from Fig. 3 it is clear that representation of chemical bond regions by DORI is not as satisfactory as IRI, namely the DORI isosurfaces corresponding to different bonds are merged together rather than separated. According to Fig. S1, some of the above problems can be relieved by adjusting DORI isovalue appropriately, but not all of them can be perfectly solved. For example, increasing the isovalue to 0.98 can largely suppress the occurrence of the noisy DORI isosurfaces between the hydrogens, however each C-C bond in this case will be weirdly represented by three small isosurfaces, which severely conflicts with common chemical intuition.

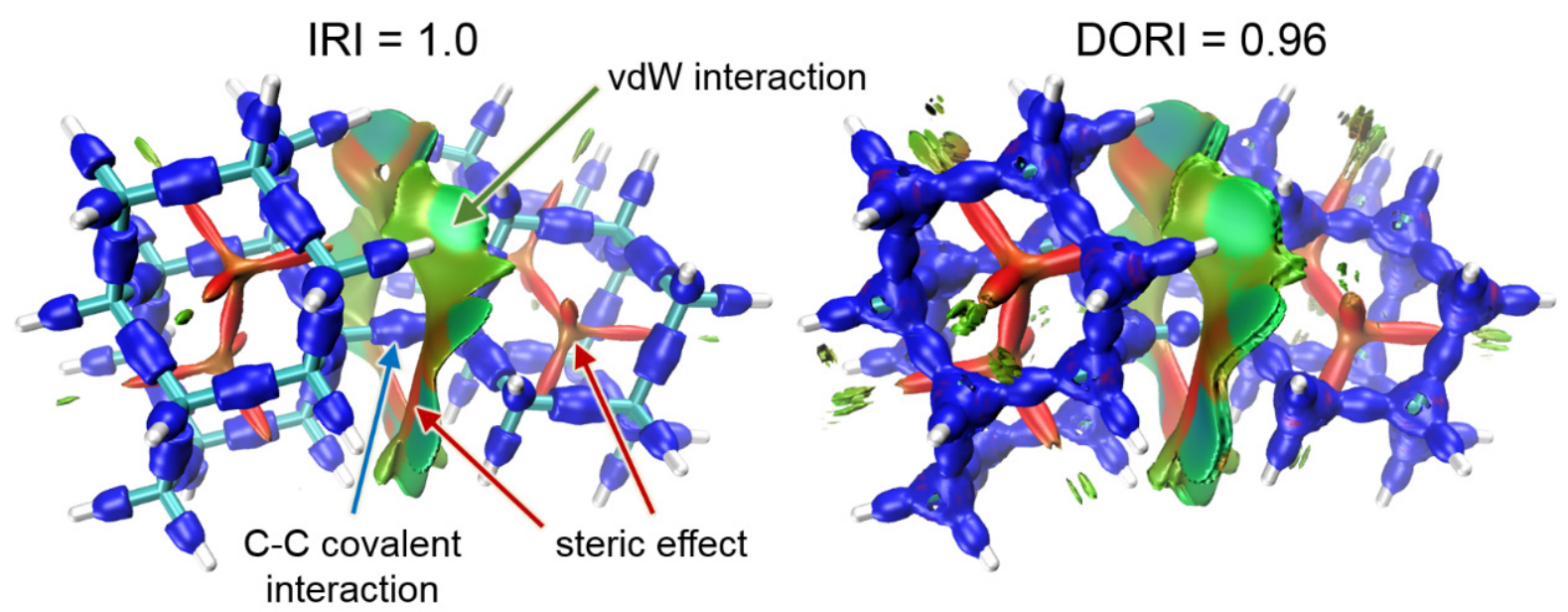

Fig. 3 Isosurface maps of IRI $=1.0$ and DORI $=0.96 . \operatorname{sign}\left(\lambda_{2}\right) \rho$ is mapped on the isosurfaces according to coloring method of Fig. 1. Grid spacing for both IRI and DORI is 0.12 Bohr. 
Another system for comparing IRI and DORI is guanine-cytosine dimer, this is a planar system and there are three H-bonds between the two molecules. Isosurface maps and color-filled plane maps of IRI and DORI are shown in Fig. 4. From Fig. 4(a) it is seen that IRI can well reveal the region corresponding to each chemical bond, and the three H-bonds can also be clearly visualized. In contrast, different chemical bonds cannot be sufficiently distinguished by the DORI isosurfaces in Fig. 4(b). In order to more fully exhibit different characters of IRI and DORI, their color-filled maps are plotted in the dimer plane, see Figs. 4(c) and 4(d), respectively. It is found that IRI varies smoothly, this is why its isosurfaces always look smooth. The variation behavior of DORI is quite complicated and it oscillates dramatically in some regions, this phenomenon directly corresponds to the frequent observation of noisy and discrete DORI isosurfaces in many situations.
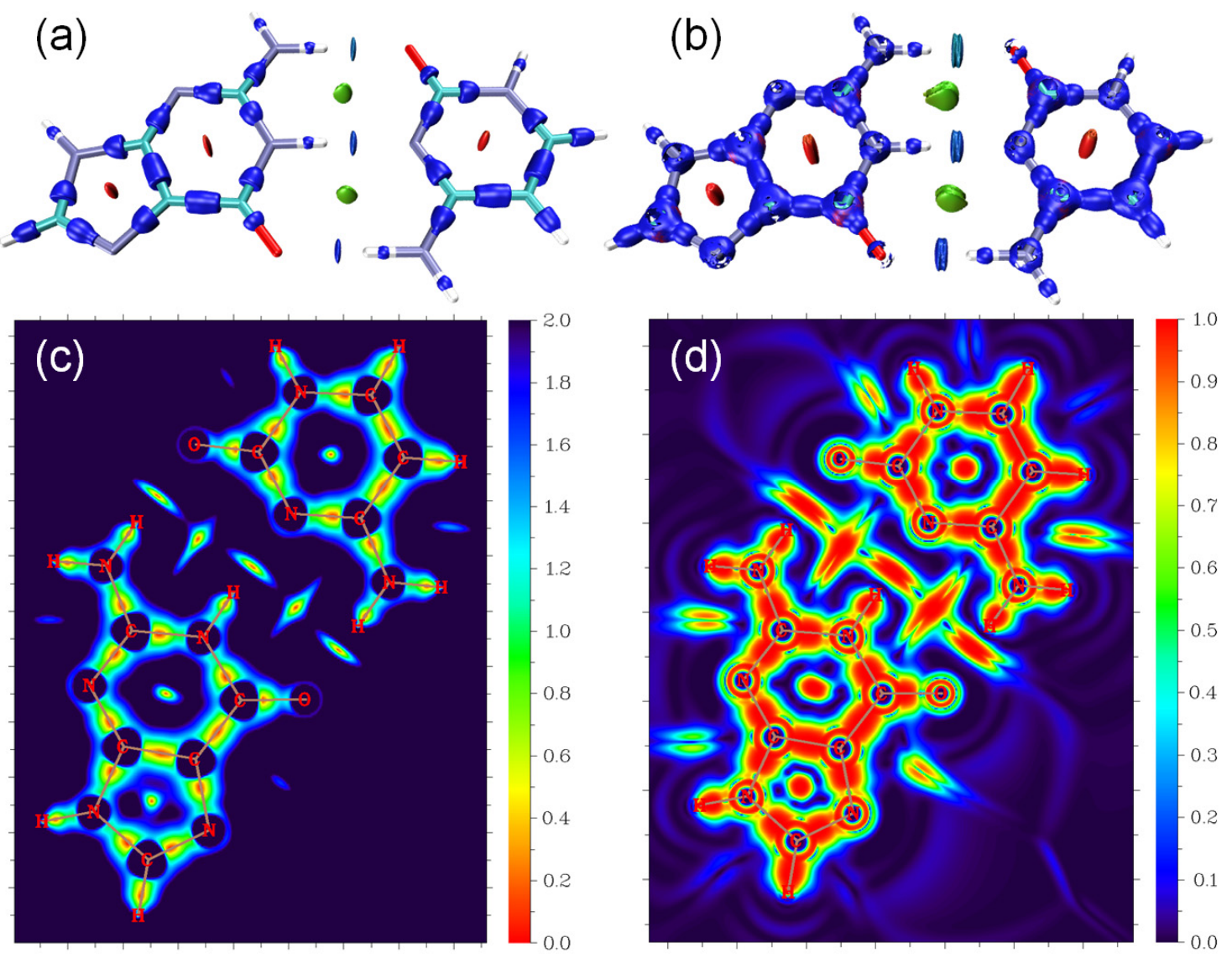

Fig. 4 Guanine-cytosine dimer. (a) IRI $=1.0$ isosurface map (b) DORI $=0.96$ isosurface map 
(c) IRI on the dimer plane (d) DORI the on dimer plane

\subsection{Relationship between IRI map and AIM topology analysis}

In the popular Bader's atoms-in-molecules (AIM) theory, ${ }^{[15,16]}$ topology analysis is often carried out for electron density to search for its critical points (CPs), which satisfy condition of $|\nabla \rho|=0$ and will be referred to as AIM critical points (AIM CPs) later. The $(3,-1)$ type of AIM CP is also known as bond critical point (BCP) and frequently employed to characterize chemical bonds and attractive weak interactions, ${ }^{[17-21]}$ while $(3,+1)$ and $(3,+3)$ types of AIM CPs correspond to ring critical point $(\mathrm{RCP})$ and cage critical point $(\mathrm{CCP})$, which are related to steric effect in small rings and cages, respectively. IRI has a close relationship with the AIM topology analysis, since both of them are able to provide insights into interatomic interactions in a chemical system. From a visual perspective, IRI map is more intuitive and useful because AIM topology analysis only represents the interactions as discrete points. Commonly, a region encompassed by an IRI isosurface also contains one or more corresponding AIM CPs, however, it is important to recognize that IRI analysis should never be simply regarded as a visual enhancement or extension of the AIM representation, because IRI has capacity of revealing some important interactions that invisible from AIM CPs. To illustrate this point, $\mathrm{Ni}\left(\mathrm{NH}_{3}\right)_{2}(\mathrm{OH})_{2}$ is taken as an example.

From Fig. 5(b) it can be seen that the two kinds of coordinate bonds in $\mathrm{Ni}\left(\mathrm{NH}_{3}\right)_{2}(\mathrm{OH})_{2}$, namely $\mathrm{Ni}-\mathrm{O}$ and $\mathrm{Ni}-\mathrm{N}$, are equally well represented by the blue isosurfaces of IRI $=1.0$. At the same time, IRI enables chemists to easily identify the prominent intramolecular H-bonds between the coordinated ammonias and hydroxyl ions. The scatter map between RDG and $\operatorname{sign}\left(\lambda_{2}\right) \rho$ is often discussed in RDG analysis, ${ }^{[1]}$ a similar plot between IRI and $\operatorname{sign}\left(\lambda_{2}\right) \rho$ for the $\mathrm{Ni}\left(\mathrm{NH}_{3}\right)_{2}(\mathrm{OH})_{2}$ is shown in Fig. 5(a), which is useful in understanding distribution character of IRI. The point at bottom of each spike in the scatter map corresponds to an IRI minimum. If the current isovalue is set to be larger than the IRI value at an IRI minimum, then an isosurface of IRI will 
occur around it. The points intersecting with the red dashed line $(I R I=1.0)$ in the scatter map correspond to the grid points constituting the isosurfaces shown in Fig. (b). In order to facilitate the understanding, the correspondence between IRI isosurfaces and the included IRI minima have been indicated by arrows between Figs. 5(a) and 5(b). Fig. 5(c) shows gradient lines of $\rho$, BCPs are shown as blue points and corresponding bond paths are drawn as brown lines. It can be seen that no AIM CP occurs in the intramolecular H-bond regions because of nonvanishing $|\nabla \rho|$, this is also why the corresponding IRI spikes pointed by the green arrows in Fig. 5(a) did not fully reach the bottom of the scatter map. This observation reflects the severe limitation of AIM theory in exhibiting interatomic interactions compared with IRI. This issue of AIM analysis is known in some systems containing intramolecular H-bonds and can be overcome by using RDG analysis instead. ${ }^{[22]}$ Obviously, the advantage of RDG is inherited by IRI due to their similar expressions.

(a)

(b)
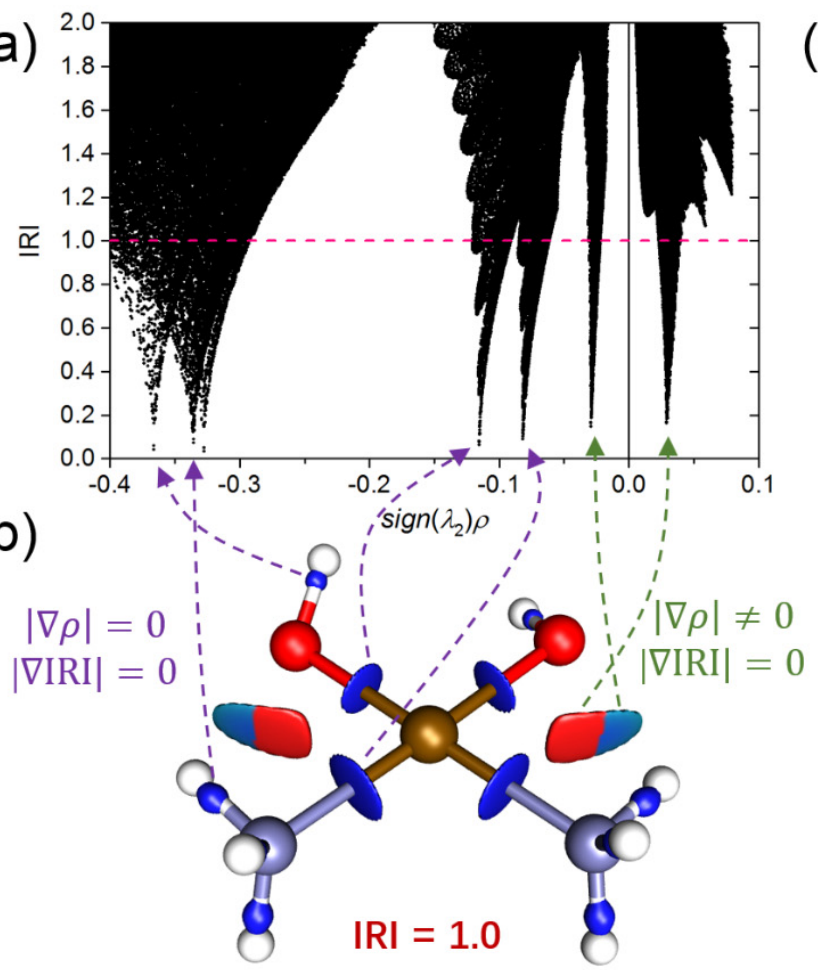

(c)

Gradient line and contour map of $\rho$

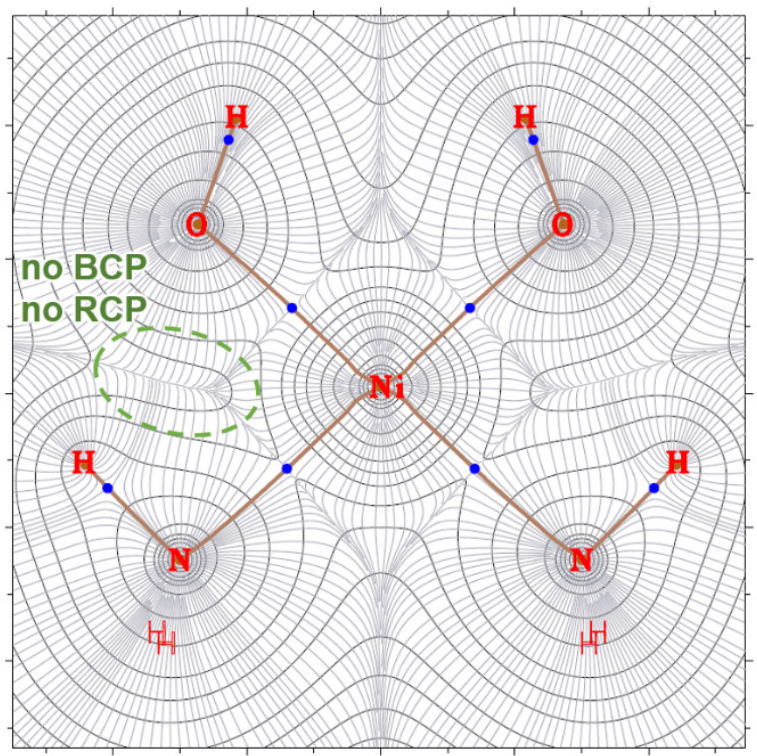

Fig. 5 Some maps of $\mathrm{Ni}\left(\mathrm{NH}_{3}\right)_{2}(\mathrm{OH})_{2}$ (a) Scatter map between IRI and $\operatorname{sign}\left(\lambda_{2}\right) \rho($ b) Isosurface map of IRI $=1.0$ (c) Gradient line map with contour lines of $\rho$ on molecular plane plotted by Multiwfn program. Blue points correspond to BCPs, brown lines represent bond paths. 
Let us look at the conditions when an IRI CP occurs to deeply understand why IRI minima and corresponding isosurfaces can reveal more interactions than AIM CPs. The point satisfying $|\nabla I R I|=0$ will be referred to as IRI CP, which requires that $\partial \mathrm{IRI} / \partial x=$ $0, \partial \mathrm{IRI} / \partial y=0$ and $\partial \mathrm{IRI} / \partial z=0$ are simultaneously met. After some mathematical derivations (see Section S1), we arrive the following relationships, where $a$ is the IRI parameter in Eq. 4.

$$
\begin{aligned}
& \frac{\partial \text { IRI }}{\partial x}=0 \Rightarrow\left(\frac{\partial \rho}{\partial x} \frac{\partial^{2} \rho}{\partial x^{2}}+\frac{\partial \rho}{\partial y} \frac{\partial^{2} \rho}{\partial x y}+\frac{\partial \rho}{\partial z} \frac{\partial^{2} \rho}{\partial x z}\right)=a \frac{|\nabla \rho|^{2}}{\rho} \frac{\partial \rho}{\partial x} \\
& \frac{\partial \text { IRI }}{\partial y}=0 \Rightarrow\left(\frac{\partial \rho}{\partial x} \frac{\partial^{2} \rho}{\partial x y}+\frac{\partial \rho}{\partial y} \frac{\partial^{2} \rho}{\partial y^{2}}+\frac{\partial \rho}{\partial z} \frac{\partial^{2} \rho}{\partial y z}\right)=a \frac{|\nabla \rho|^{2}}{\rho} \frac{\partial \rho}{\partial y} \\
& \frac{\partial \text { IRI }}{\partial z}=0 \Rightarrow\left(\frac{\partial \rho}{\partial x} \frac{\partial^{2} \rho}{\partial x z}+\frac{\partial \rho}{\partial y} \frac{\partial^{2} \rho}{\partial y z}+\frac{\partial \rho}{\partial z} \frac{\partial^{2} \rho}{\partial z^{2}}\right)=a \frac{|\nabla \rho|^{2}}{\rho} \frac{\partial \rho}{\partial z}
\end{aligned}
$$

The above three conditions are automatically satisfied at AIM CPs, where $\partial \rho / \partial x=0$, $\partial \rho / \partial y=0, \partial \rho / \partial z=0$ and thus $|\nabla \rho|=0$, so every AIM CP exactly corresponds to a IRI minimum with IRI $=0$, this is why IRI isosurfaces always appear around AIM CPs. However, IRI minima and accompanied isosurfaces are capable of revealing more interactions because some other points may also satisfy all above conditions. To more easily elucidate this point, it is noticed that the three Cartesian axes $(x, y, z)$ can be rotated so that the three eigenvectors of Hessian matrix of $\rho$ happen to be parallel to the three new Cartesian axes $\left(x^{\prime}, y^{\prime}, z^{\prime}\right)$, then the non-diagonal terms of the Hessian will be eliminated, in this case Eq. 5 simplifies to

$$
\begin{aligned}
& \frac{\partial \rho}{\partial x^{\prime}} \frac{\partial^{2} \rho}{\partial x^{\prime 2}}=a \frac{|\nabla \rho|^{2}}{\rho} \frac{\partial \rho}{\partial x^{\prime}} \\
& \frac{\partial \rho}{\partial y^{\prime}} \frac{\partial^{2} \rho}{\partial y^{\prime 2}}=a \frac{|\nabla \rho|^{2}}{\rho} \frac{\partial \rho}{\partial y^{\prime}} \\
& \frac{\partial \rho}{\partial z^{\prime}} \frac{\partial^{2} \rho}{\partial z^{\prime 2}}=a \frac{|\nabla \rho|^{2}}{\rho} \frac{\partial \rho}{\partial z^{\prime}}
\end{aligned}
$$

Now it is easy to recognize that IRI CP occurs under more relaxed conditions than AIM CP. For example, IRI CP also occurs at a point where

$$
\frac{\partial^{2} \rho}{\partial x^{\prime 2}}=a \frac{|\nabla \rho|^{2}}{\rho} \quad \frac{\partial \rho}{\partial y^{\prime}}=0 \quad \frac{\partial \rho}{\partial z^{\prime}}=0
$$


apparently the requirement $\partial \rho / \partial x^{\prime}=0$ of AIM CP no longer has to be satisfied in this case. Our numerical examination verified that the IRI minima enclosed by the IRI isosurfaces revealing intramolecular H-bonds in Fig. 5 (b) actually correspond to this kind of situation, see Section S2 for intuitive illustration and more discussion.

We noted that occurrence conditions of RDG CPs has been discussed in Ref. [23], however we found the condition given in that work, namely the Eq. 4 in Ref. [23], is incorrect. Actual occurrence conditions of RDG CPs correspond to Eq. 5 or 6 with $a=$ $4 / 3$.

\section{Some examples and applications of IRI}

Next, some application examples of IRI will be given to demonstrate its strong ability of exhibiting interactions in a wide variety of chemical systems.

Fig. 5 presents four chemical systems, which not only contain chemical bonds but also involve intramolecular or intermolecular interactions. In the case of $\mathrm{N}($ phenyl) 3 , it can be seen that IRI nicely exhibits the interaction between each pair of phenyl groups, the region close to the nitrogen atom shows partial steric effect since the mapped color is orange, while the green part of the IRI isosurfaces implies attractive dispersion effect. In the $\left(\mathrm{NO}_{2}\right)_{2} \mathrm{C}-\mathrm{C}\left(\mathrm{NH}_{2}\right)_{2}$ molecule, which is also known as FOX-7, an energetic compound, the H-bonds between the nitro groups and amino groups can be clearly visualized, while the steric region due to their close contact is also evident from the red isosurfaces. The $\mathrm{Cl}_{5} \mathrm{At} \cdots \mathrm{SH}_{2}$ halogen bond dimer was studied recently by us, ${ }^{[24]}$ the At$\mathrm{Cl}$ bonding regions are nicely revealed by the corresponding blue IRI isosurfaces, indicating that IRI also works for very heavy elements. The IRI isosurface corresponding to the halogen bond between the $\mathrm{Cl}_{5} \mathrm{At}$ and $\mathrm{SH}_{2}$ molecules shows blue color, suggesting their high binding strength. This observation is in line with the large binding energy obtained by quantum chemistry calculation. ${ }^{[24]}$ IRI can also well reveal ionic bonds. As shown in the case of $\mathrm{C}_{6} \mathrm{H}_{5} \mathrm{OONa}$, the strong electrostatic attractive interaction between the oxygens of the $\mathrm{C}_{6} \mathrm{H}_{5} \mathrm{OO}^{-}$anion and $\mathrm{Na}^{+}$cation is exhibited by 
the two blue parts of the IRI isosurface.

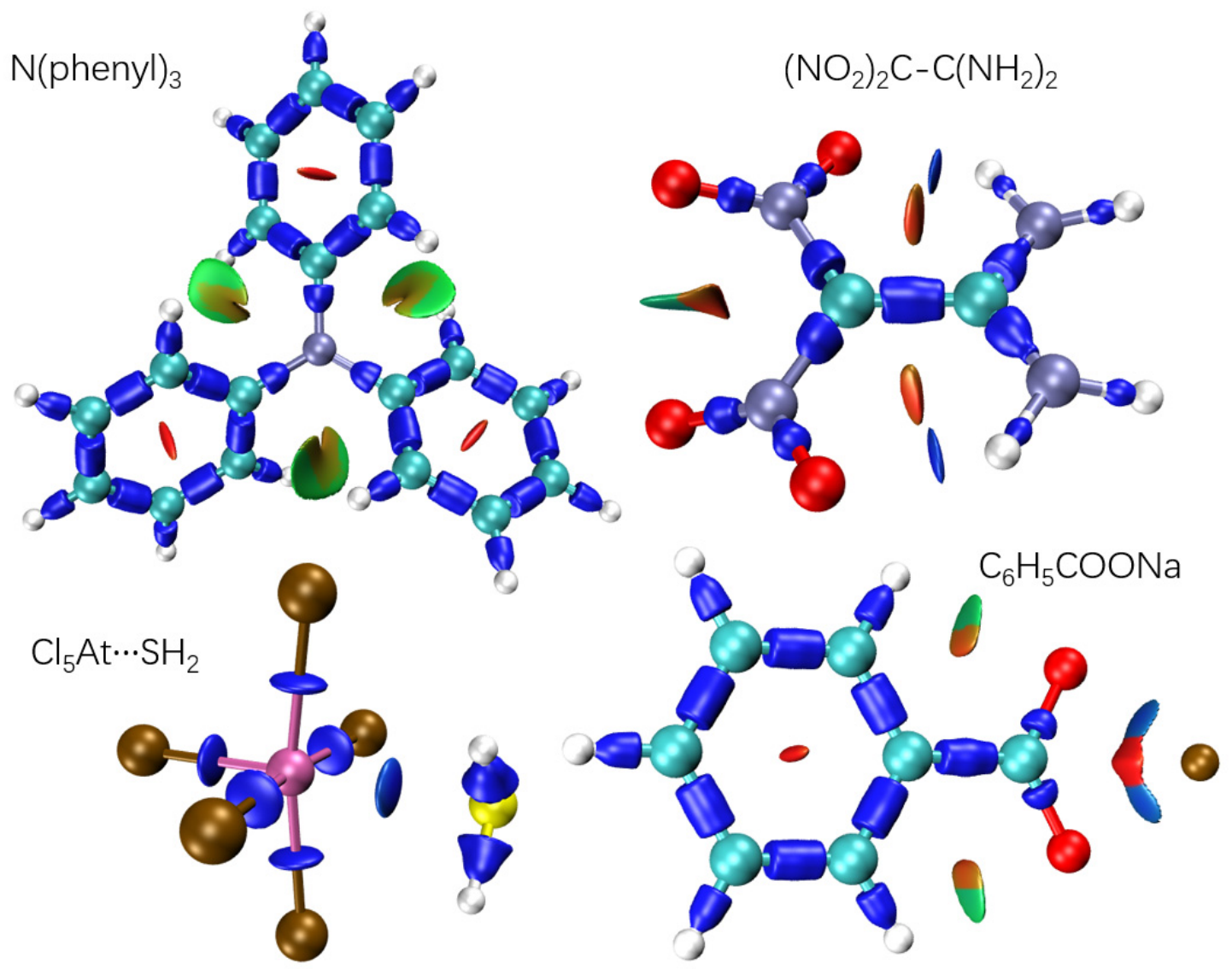

Fig. 6 Isosurface map of IRI of four systems. $\operatorname{sign}\left(\lambda_{2}\right) \rho$ is mapped on the isosurfaces according to coloring method of Fig. 1. Isovalue of IRI is chosen to be 1.0 except for $\mathrm{Cl}_{5} \mathrm{At}$...SH $\mathrm{SH}_{2}$, which employs IRI of 0.9 to gain better graphical effect. def2-TZVP basis set ${ }^{[25]}$ was employed for At in the calculation.

To further examine the universality of IRI, we also considered some transition metal complexes, their IRI isosurface maps are shown in Fig. 6. The coordinate bonds between the $\mathrm{Ru}$ and coordinated nitrogen atoms in $\mathrm{Ru}(\mathrm{bpy})_{3}$ are clearly exhibited by blue IRI isosurfaces. The vdW interaction within in each bpy ligand and that between different bpy ligands are also clearly revealed by the corresponding IRI isosurfaces. In $\left[\mathrm{Re}_{2} \mathrm{Cl}_{8}\right]^{2-}$, IRI isosurfaces revealed the $\mathrm{Re}-\mathrm{Cl}$ and $\mathrm{Re}-\mathrm{Re}$ bonds, the vdW interaction due to the close contact between the ligand chlorine atoms can also be detected from the IRI map. Iron atom in ferrocene forms chemical bond to each of coordinated carbons 
in the two cyclopentadienyl rings, this point is well represented by the blue regions of IRI isosurfaces in Fig. 6. The steric effect in the five-membered carbon rings and the $\mathrm{Fe}-\mathrm{C}-\mathrm{C}$ rings can be identified by the red areas of the isosurfaces.
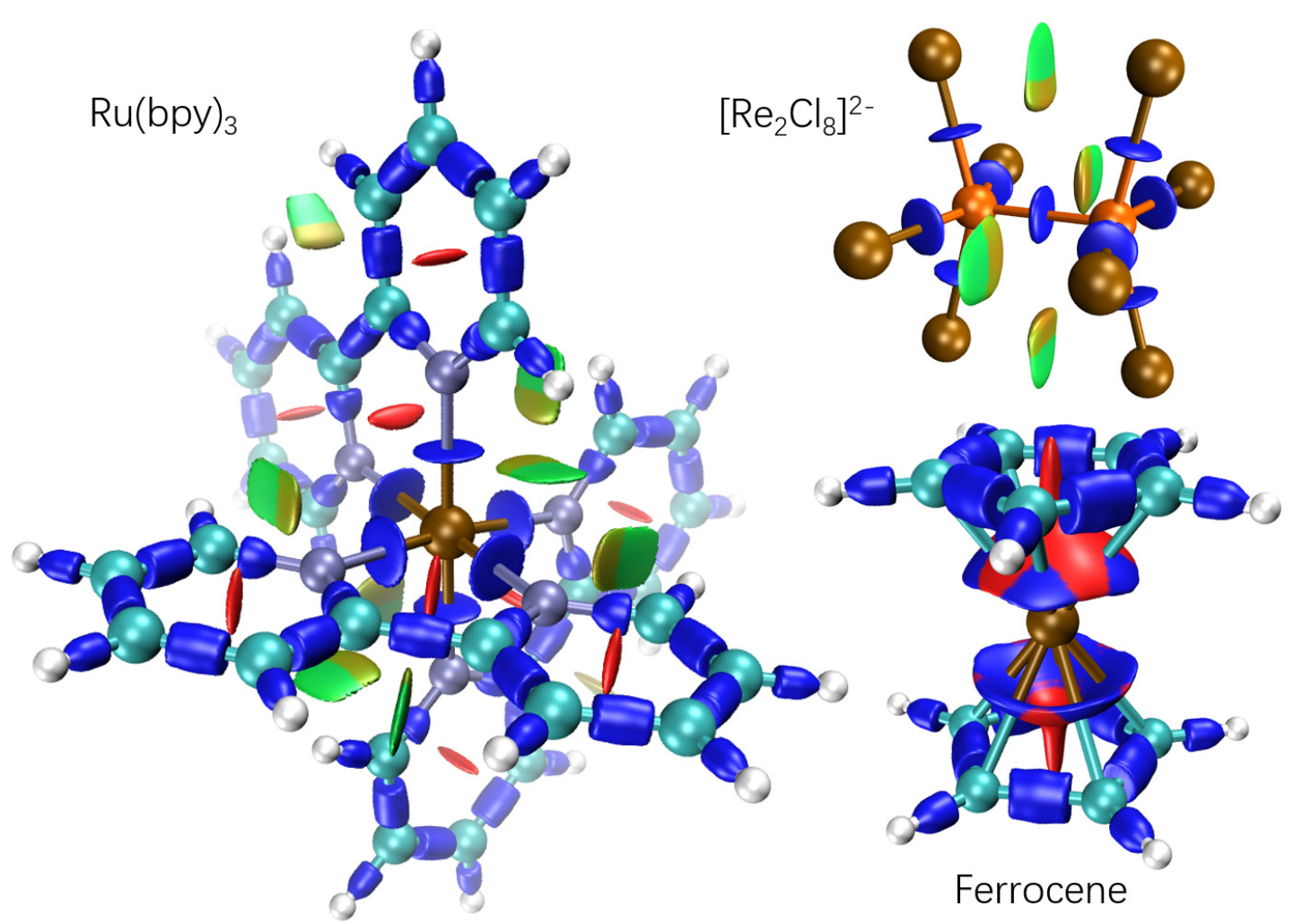

Ferrocene

Fig. 7 Isosurface map of IRI of three transition metal complexes. $\operatorname{sign}\left(\lambda_{2}\right) \rho$ is mapped on the isosurfaces according to coloring method of Fig. 1. In order to obtain most ideal graphical effect, isovalues of IRI for $\mathrm{Ru}(\text { bpy })_{3},\left[\mathrm{Re}_{2} \mathrm{Cl}_{8}\right]^{2-}$ and ferrocene are set to be $0.95,0.9$ and 1.0, respectively.

IRI is very useful in studying chemical reactions since smooth transition between weak interactions and chemical bonds can be ideally revealed by IRI analysis. As an example, the IRI maps at representative points of IRC (intrinsic reaction coordinate) path of Diels-Alder addition between cis-1,3-butadiene and ethene are shown in Fig. 9. The first point of this path corresponds to reaction complex, from the distribution and color of the IRI isosurfaces it can be seen that in this geometry there is evident vdW interaction between the two molecules and within the butadiene. As the reaction goes on from reactants to transition state (TS), the C-C bonds to be formed are gradually 
strengthened, and the electron density in the bonding regions is also gradually accumulated. Therefore, it can be seen that the color of the IRI isosurfaces in the corresponding regions become more and more blue. Meanwhile, the steric effect in the ring consisting of the six carbons becomes more and more prominent, this is why the corresponding isosurface gradually changes from green to orange. After passing the TS, the IRI isosurfaces of the new $\mathrm{C}-\mathrm{C}$ bonds gradually elongate along the bonding direction, implying the character of the bonds becomes more and more like that of an ordinary $\mathrm{C}-\mathrm{C}$ bond. The animation file showing full dynamic variation of IRI along this IRC process has been given in supplemental material.

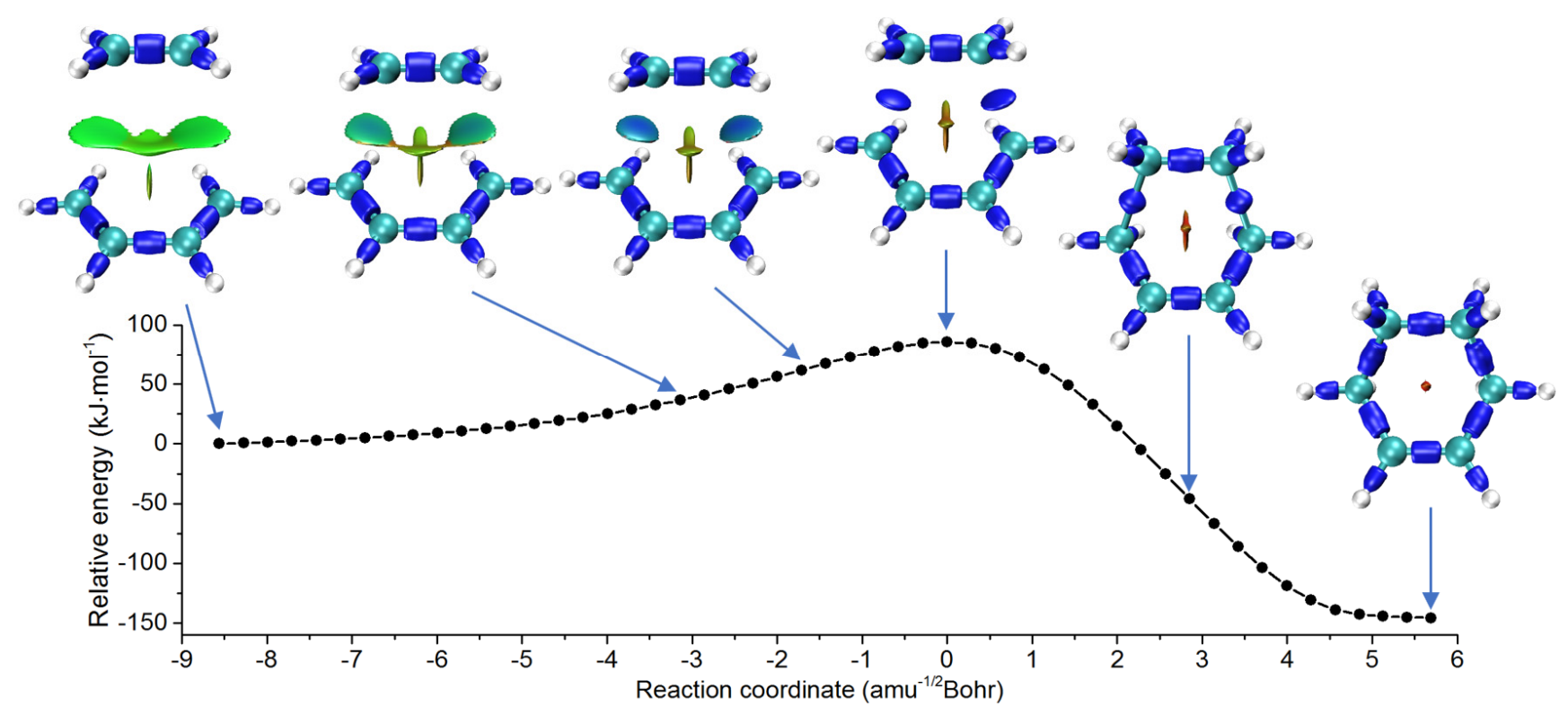

Fig. 8 Variation of isosurface map of IRI $=0.95$ during Diels-Alder addition between $c i s-1,3-$ butadiene and ethene. $\operatorname{sign}\left(\lambda_{2}\right) \rho$ is mapped on the isosurfaces according to coloring method of Fig. 1 .

Another example illustrating the capacity of IRI in visually studying chemical reaction is the $\mathrm{OH}^{-}+\mathrm{CH}_{3} \mathrm{CH}_{2} \mathrm{Br} \rightarrow \mathrm{CH}_{3} \mathrm{CH}_{2} \mathrm{OH}+\mathrm{Br}^{-} \mathrm{SN}_{2}$ substitution reaction, IRI isosurfaces at some representative points in the IRC path are given in Fig. 9. From the isosurfaces at the first point, it can be seen that $\mathrm{OH}^{-}$forms weak $\mathrm{H}$-bonds with the $\mathrm{CH}_{3} \mathrm{CH}_{2} \mathrm{Br}$ molecule. As the reaction proceeds, IRI isosurfaces indicate that the $\mathrm{O} \ldots \mathrm{CH}_{2}$ 
noncovalent interaction gradually transitions to a typical $\mathrm{O}-\mathrm{C}$ covalent bond, while the old $\mathrm{C}-\mathrm{Br}$ bond gradually breaks and the electron density in the bonding region correspondingly decreases. At the final stage, the leaving $\mathrm{Br}^{-}$anion is stabilized by the $\mathrm{CH}_{3} \mathrm{CH}_{2} \mathrm{OH}$ via vdW attractive interaction, as revealed by the broad green isosurface of IRI. The animation file displaying the whole variation of IRI isosurfaces in this example can be found in supplemental material.

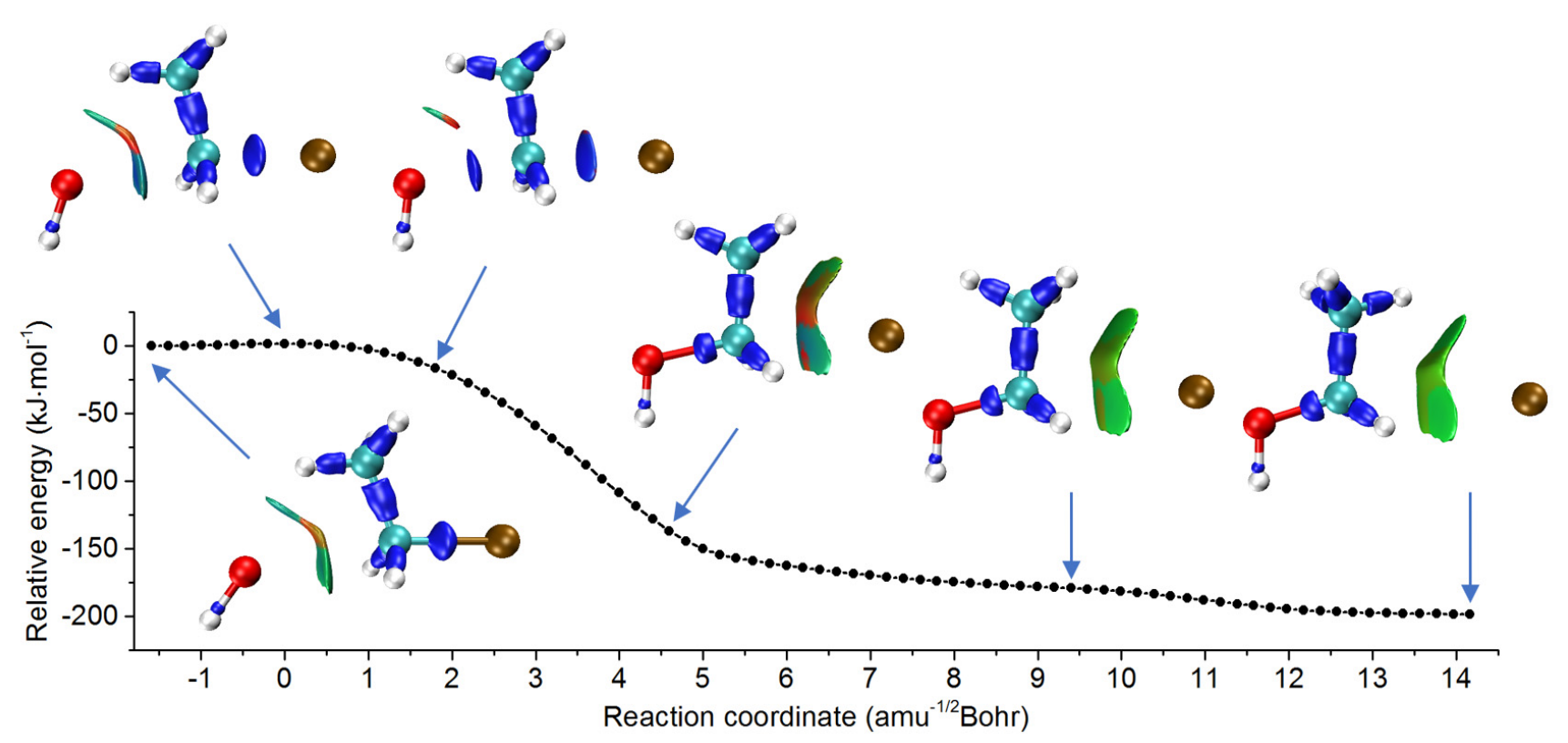

Fig. 9 Variation of isosurface map of IRI $=0.95$ during $\mathrm{SN}_{2}$ substitution reaction of $\mathrm{OH}^{-}+$ $\mathrm{CH}_{3} \mathrm{CH}_{2} \mathrm{Br} \rightarrow \mathrm{CH}_{3} \mathrm{CH}_{2} \mathrm{OH}+\mathrm{Br}^{-}$. $\operatorname{sign}\left(\lambda_{2}\right) \rho$ is mapped on the isosurfaces according to coloring method of Fig. 1.

It is worth to note that the success of applying IRI analysis on IRC paths implies that it is very promising to employ IRI in characterizing variation of covalent and noncovalent interactions in ab initio molecular dynamics simulations, this possibility will be explored in the future. 


\section{IRI- $\pi$ : A function exhibiting $\pi$ interactions}

Inspired by LOL- $\pi,{ }^{[26]}$ which has been very popular in revealing favorable delocalization path of $\pi$ electron, ${ }^{[27,28]}$ here we also propose IRI- $\pi$ aiming for revealing $\pi$ interaction regions. The only difference between IRI- $\pi$ and IRI is that the former only considers $\pi$ orbitals when calculating the electron density and its gradient involved in IRI. Since recently we have proposed an efficient, robust and blackbox algorithm for automatically identifying $\pi$ orbitals, ${ }^{[29]}$ and it has been implemented into Multiwfn program, evaluation of IRI- $\pi$ for practical $\pi$-conjugated systems can be easily achieved.

We will take a few examples to examine the character of IRI- $\pi$. Since steric effect is not related to the present discussion, $\rho$ instead of $\operatorname{sign}\left(\lambda_{2}\right) \rho$ will be mapped on the IRI- $\pi$ isosurfaces. Figs. 9(a) and 9(b) show isosurface maps of IRI- $\pi=1.0$ for ethene and acetylene, respectively. It can be seen that for a typical double bond, isosurfaces of IRI- $\pi$ symmetrically occur above and below the bond, while for a typical triple bond, which include two sets of $\pi$ interactions, the isosurface shows torus shape. Due to the very different shapes of the IRI- $\pi$ isosurfaces for different types of bonds, it is possible to employ IRI- $\pi$ to distinguish natures of conjugated bonds. We take the bonds in the cyclo[18]carbon as an example to illustrate this point. This molecule was recently observed in condensed phase and attracted wide attentions due to its unusual electronic and geometric structures, and we have made comprehensive investigations on this unusual system and its analogues, and it was found that short and long $\mathrm{C}-\mathrm{C}$ bonds occur alternatively in the cyclo[18]carbon and they show markedly different characters and strengths. ${ }^{[27,30-33]}$. Fig. 9(c) displays IRI- $\pi$ isosurface map of the cyclo[18]carbon, it can be seen that both kinds of bonds have torus-shaped isosurfaces, so they both show double $\pi$-interaction characteristics. However, their difference is also distinct. The IRI$\pi$ isosurfaces around the short $\mathrm{C}-\mathrm{C}$ bonds look visibly more blue than those around the long $\mathrm{C}-\mathrm{C}$ bonds, indicating that $\pi$ electrons distribute more densely on the short $\mathrm{C}-\mathrm{C}$ bonds and thus the short $\mathrm{C}-\mathrm{C}$ bonds must correspond to relatively stronger $\pi$ interaction. The conclusion drawn from visualizing IRI- $\pi$ is in line with ELF and valence electron density analyses. ${ }^{[27]}$ 


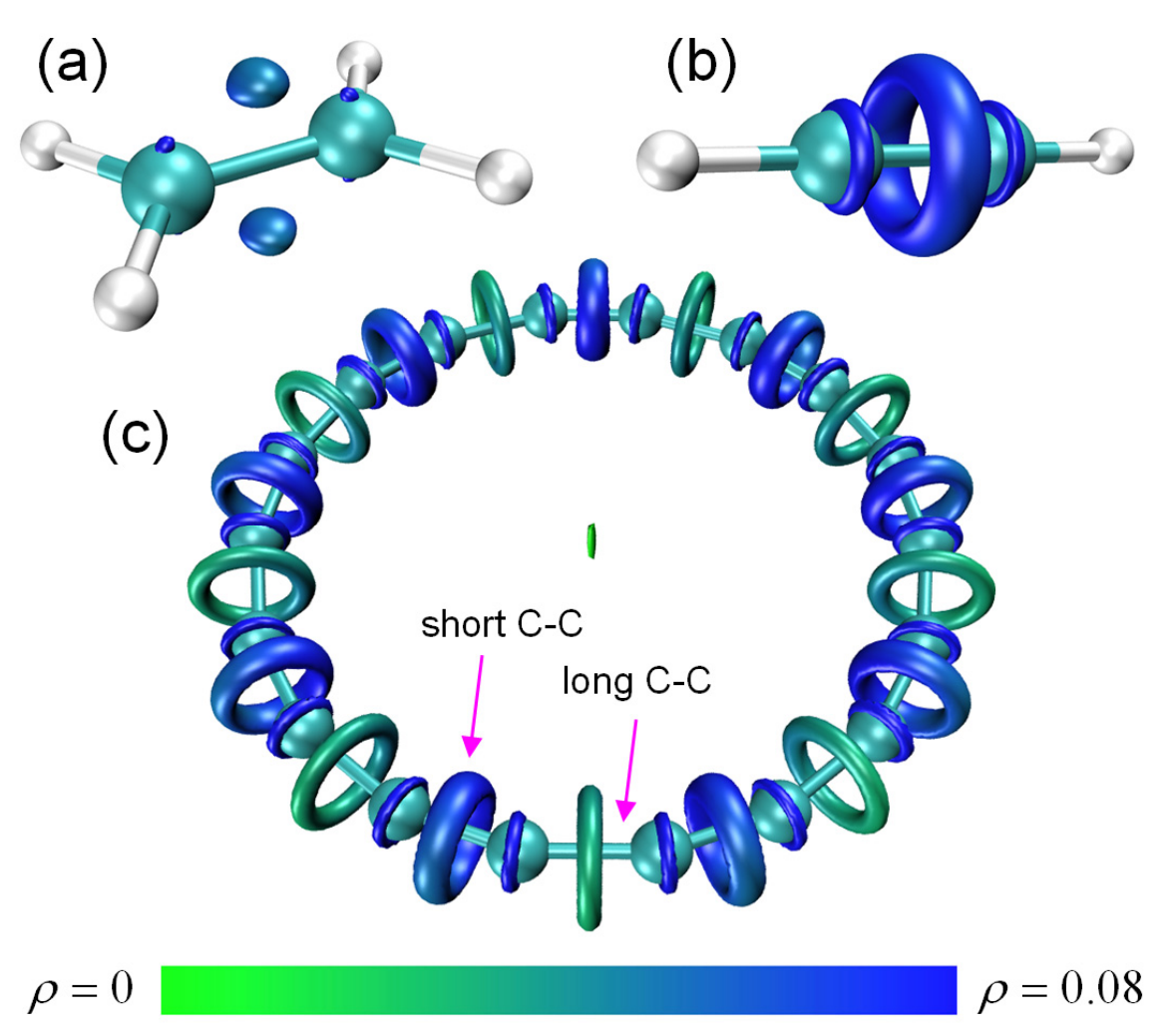

Fig. 10 Isosurface map of IRI- $\pi=1.0$ of (a) ethene (b) acetylene (c) cyclo[18]carbon. Electron density is mapped on the isosurfaces according to the color bar. The wavefunction of cyclo[18]carbon was taken from Ref. [27] 


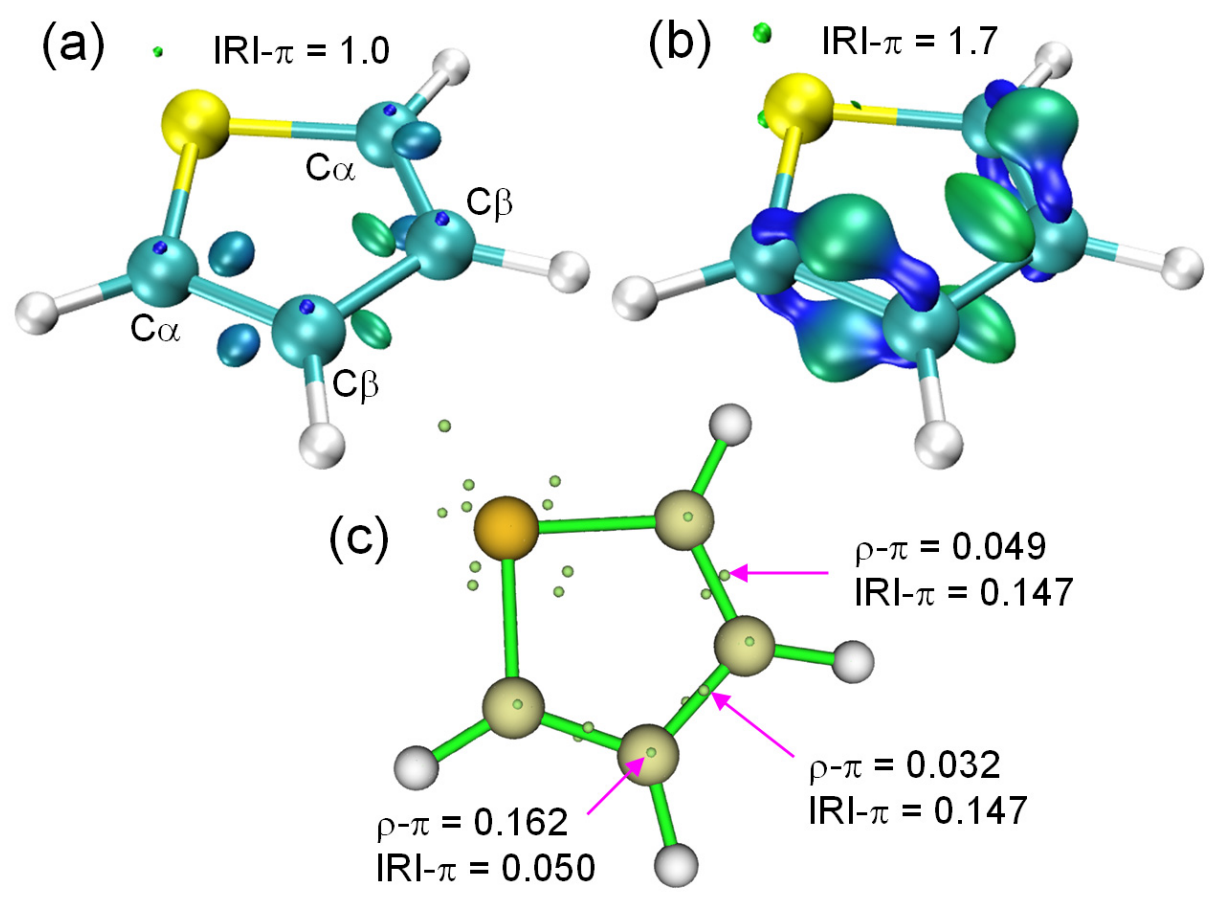

Fig. 11 IRI- $\pi$ of thiophene. (a) Isosurface map of IRI- $\pi=1.0$ (a) Isosurface map of IRI- $\pi=1.7$ Coloring method is identical Fig. 9 (c) Minima of IRI- $\pi$. Electron density of $\pi$ electron and IRI$\pi$ values are labelled on some of them

Another example illustrating the value of IRI- $\pi$ analysis is thiophene, see Fig. 11. In this analysis, only the Pipek-Mezey localized molecular orbitals ${ }^{[34]}$ of $\pi$-type were considered in the calculation of IRI- $\pi$. It is well-known that the four carbons in this molecule show $\pi$-conjugation character, not only the distribution of the IRI isosurfaces in Fig. 11(a) reflects this point, but also the mapped colors indicate that the $\pi$-interaction of the $C_{\alpha}-C_{\beta}$ bonds is stronger than that of the $C_{\beta}-C_{\beta}$ bond. If rising the IRI- $\pi$ isovalue to 1.7, as shown in Fig. 11(b), the distribution range of the isosurfaces will become significantly wider. In this case, it is interestingly found that the IRI- $\pi$ isosurfaces above and below each $\mathrm{C}_{\alpha}-\mathrm{C}_{\beta}$ bond have connected to the two bonding atoms, while the isosurface corresponding to the $\mathrm{C}_{\beta}-\mathrm{C}_{\beta}$ bond is still fully isolated from the atoms. Our investigations on a large number of systems via IRI- $\pi$ showed that this is a common phenomenon rather than a special case. Apparently, this special character of IRI- $\pi$ enables one to differentiate $\pi$-interaction strengths even by solely inspecting the shape 
of IRI- $\pi$ isosurfaces at an appropriate isovalue. The grid-based basin analysis module in Multiwfn code is very universal, it is capable of locating maxima or minima of any real space function, hence allowing the analysis of the IRI- $\pi$ at quantitative level. Fig. 11(c) exhibits position of all minima of IRI- $\pi$, the $\pi$ electron density $(\rho-\pi)$ and IRI- $\pi$ value of some representative minima are also labelled. It can be seen that $\rho-\pi$ at the IRI- $\pi$ minima corresponding to the $\mathrm{C}_{\alpha}-\mathrm{C}_{\beta}$ bonds is distinctly higher than the counterpart of the $\mathrm{C}_{\beta}-\mathrm{C}_{\beta}$ bond, further exhibiting the difference in their $\pi$-interaction strengths. The examples in this section imply the great value of applying IRI- $\pi$ in the study of $\pi$ interactions.

\section{Summary}

In this work, a new real space function named IRI is proposed aiming for visually reveal both chemical bonds and weak interactions in chemical systems. Compared to the popular RDG, the advantage of IRI is that it is able to exhibit all kinds of interactions in an equal footing. IRI is preferred over DORI, which is another function having similar purpose, not only because IRI has a much simpler expression and thus easier to calculate, but also IRI isosurface has a conspicuously better graphical effect. A batch of examples of IRI have demonstrated that IRI has a strong universality and practicability in exhibiting interatomic interactions in a wide range of chemical systems, and it can provide a smooth representation of variation of bonding during a chemical reaction. It is noteworthy that IRI is simply dependent of electron density and its gradient, therefore it can be evaluated not only based on the electron density generated by quantum chemistry calculation, but also based on high-resolution electron density data from Xray diffraction experiment. In addition, we propose IRI- $\pi$, which is dedicated to characterize $\pi$ interaction based on $\pi$ electron density. A few examples showed that $\operatorname{sign}\left(\lambda_{2}\right) \rho$ mapped IRI- $\pi$ isosurfaces are capable of distinguishing types of $\pi$ interactions and differentiate $\pi$-interaction strengths.

IRI and IRI- $\pi$ have been efficiently implemented in our freely available code 
Multiwfn, which is a highly-integrated wavefunction analysis software and compatible with electronic wavefunctions produced by wide variety of quantum chemistry programs. A tutorial of performing various kinds of IRI analyses in Multiwfn can be found in supplemental material. We hope IRI and IRI- $\pi$ will become new popular tools for computational chemists to study chemical problems.

\section{Funding information}

This research was not funded.

\section{CRediT authorship contribution statement}

Tian Lu: Conceptualization, Software, Investigation, Writing - Original Draft, Visualization

Qinxue Chen: Writing - Review \& Editing, Investigation

\section{Conflicts of interest}

The authors declare no conflict of interest.

\section{Supplemental material}

Tutorial of performing IRI analysis in Multiwfn; animation files showing bonding variation of Diels-Alder addition and $\mathrm{SN}_{2}$ substitution reactions; derivation of conditions of occurrence of IRI critical points; Illustrating why IRI minima corresponding to intramolecular $\mathrm{H}-$ bond in $\mathrm{Ni}\left(\mathrm{NH}_{3}\right)_{2}(\mathrm{OH})_{2}$ can occur; isosurface maps of DORI of diamantane-diamantane at different isovalues.

\section{References}

1. E. R. Johnson; S. Keinan; P. Mori-Sánchez; J. Contreras-García; A. J. Cohen; W. Yang. Revealing Noncovalent Interactions. J. Am. Chem. Soc., 132, 6498-6506 (2010). DOI: 10.1021/ja100936w.

2. T. Lu; F. Chen. Meaning and Functional Form of the Electron Localization Function. Acta Phys. Chim. Sin., 27, 2786-2792 (2011). DOI: 10.3866/PKU.WHXB20112786.

3. T. Lu; Q. Chen. Revealing Molecular Electronic Structure via Analysis of Valence Electron Density. 
Acta Phys. -Chim. Sin., 34, 503-513 (2018). DOI: 10.3866/pku.Whxb201709252.

4. A. D. Becke; K. E. Edgecombe. A Simple Measure of Electron Localization in Atomic and Molecular Systems. J. Chem. Phys., 92, 5397-5403 (1990). DOI: 10.1063/1.458517.

5. N. Gillet; R. Chaudret; J. Contreras-García; W. Yang; B. Silvi; J.-P. Piquemal. Coupling Quantum Interpretative Techniques: Another Look at Chemical Mechanisms in Organic Reactions. J. Chem. Theory Comput., 8, 3993-3997 (2012). DOI: 10.1021/ct300234g.

6. P. de Silva; C. Corminboeuf. Simultaneous Visualization of Covalent and Noncovalent Interactions Using Regions of Density Overlap. J. Chem. Theory Comput., 10, 3745-3756 (2014). DOI: $10.1021 / \mathrm{ct} 500490 \mathrm{~b}$.

7. T. Lu; F. Chen. Multiwfn: A Multifunctional Wavefunction Analyzer. J. Comput. Chem., 33, 580592 (2012). DOI: 10.1002/jcc.22885.

8. W. Humphrey; A. Dalke; K. Schulten. VMD: Visual molecular dynamics. J. Mol. Graph., 14, 3338 (1996). DOI: 10.1016/0263-7855(96)00018-5.

9. M. J. Frisch; G. W. Trucks; H. B. Schlegel; G. E. Scuseria; M. A. Robb; J. R. Cheeseman; G. Scalmani; V. Barone; G. A. Petersson; H. Nakatsuji; X. Li; M. Caricato; A. V. Marenich; J. Bloino; B. G. Janesko; R. Gomperts; B. Mennucci; H. P. Hratchian; J. V. Ortiz; A. F. Izmaylov; J. L. Sonnenberg; Williams; F. Ding; F. Lipparini; F. Egidi; J. Goings; B. Peng; A. Petrone; T. Henderson; D. Ranasinghe; V. G. Zakrzewski; J. Gao; N. Rega; G. Zheng; W. Liang; M. Hada; M. Ehara; K. Toyota; R. Fukuda; J. Hasegawa; M. Ishida; T. Nakajima; Y. Honda; O. Kitao; H. Nakai; T. Vreven; K. Throssell; J. A. Montgomery Jr.; J. E. Peralta; F. Ogliaro; M. J. Bearpark; J. J. Heyd; E. N. Brothers; K. N. Kudin; V. N. Staroverov; T. A. Keith; R. Kobayashi; J. Normand; K. Raghavachari; A. P. Rendell; J. C. Burant; S. S. Iyengar; J. Tomasi; M. Cossi; J. M. Millam; M. Klene; C. Adamo; R. Cammi; J. W. Ochterski; R. L. Martin; K. Morokuma; O. Farkas; J. B. Foresman; D. J. Fox. Gaussian 16 A.03, Wallingford, CT, 2016.

10. S. Grimme; S. Ehrlich; L. Goerigk. Effect of the damping function in dispersion corrected density functional theory. J. Comput. Chem., 32, 1456-1465 (2011). DOI: 10.1002/jcc.21759.

11. P. J. Stephens; F. J. Devlin; C. F. Chabalowski; M. J. Frisch. Ab Initio Calculation of Vibrational Absorption and Circular Dichroism Spectra Using Density Functional Force Fields. J. Phys. Chem., 98, 11623-11627 (1994). DOI: 10.1021/j100096a001.

12. D. Andrae; U. Häußermann; M. Dolg; H. Stoll; H. Preuß. Energy-adjustedab initio pseudopotentials for the second and third row transition elements. Theor. Chim. Acta, 77, 123-141 (1990). DOI: 10.1007/BF01114537.

13. R. Krishnan; J. S. Binkley; R. Seeger; J. A. Pople. Self-consistent molecular orbital methods. XX. A basis set for correlated wave functions. J. Chem. Phys., 72, 650-654 (1980).

14. P. R. Schreiner; L. V. Chernish; P. A. Gunchenko; E. Y. Tikhonchuk; H. Hausmann; M. Serafin; S. Schlecht; J. E. P. Dahl; R. M. K. Carlson; A. A. Fokin. Overcoming lability of extremely long alkane carbon-carbon bonds through dispersion forces. Nature, 477, 308-311 (2011). DOI: 10.1038/nature10367.

15. F. W. Bader. Atoms in Molecules: A Quantum Theory. Oxford University Press: New York, (1994).

16. C. F. Matta; R. J. Boyd. The Quantum Theory of Atoms in Molecules - From Solid State to DNA and Drug Design. WILEY-VCH Verlag GmbH \& Co. KGaA: Weinheim, 2007.

17. S. Manzetti; T. Lu; H. Behzadi; M. D. Estrafili; H.-L. Thi Le; H. Vach. Intriguing properties of unusual silicon nanocrystals. RSC Adv, 5, 78192-78208 (2015). DOI: 10.1039/C5RA17148B.

18. S. Manzetti; T. Lu. The geometry and electronic structure of Aristolochic acid: possible implications for a frozen resonance. J. Phys. Org. Chem., 26, 473-483 (2013). DOI: 10.1002/poc.3111. 
19. T. Lu; S. Manzetti. Wavefunction and reactivity study of benzo[a]pyrene diol epoxide and its enantiomeric forms. Struct. Chem., 25, 1521-1533 (2014). DOI: 10.1007/s11224-014-0430-6.

20. F. Zhou; Y. Liu; Z. Wang; T. Lu; Q. Yang; Y. Liu; B. Zheng. A new type of halogen bond involving multivalent astatine: an ab initio study. Phys. Chem. Chem. Phys., 21, 15310-15318 (2019). DOI: 10.1039/C9CP02406A.

21. Z. Wang; Y. Liu; B. Zheng; F. Zhou; Y. Jiao; Y. Liu; X. Ding; T. Lu. A theoretical investigation on $\mathrm{Cu} / \mathrm{Ag} / \mathrm{Au}$ bonding in $\mathrm{XH} 2 \mathrm{P} \cdots \mathrm{MY}(\mathrm{X}=\mathrm{H}, \mathrm{CH} 3, \mathrm{~F}, \mathrm{CN}, \mathrm{NO} 2 ; \mathrm{M}=\mathrm{Cu}, \mathrm{Ag}, \mathrm{Au} ; \mathrm{Y}=\mathrm{F}, \mathrm{Cl}, \mathrm{Br}, \mathrm{I})$ complexes. J. Chem. Phys., 148, 194106 (2018). DOI: 10.1063/1.5027605.

22. J. R. Lane; J. Contreras-García; J.-P. Piquemal; B. J. Miller; H. G. Kjaergaard. Are Bond Critical Points Really Critical for Hydrogen Bonding? J. Chem. Theory Comput., 9, 3263-3266 (2013). DOI: $10.1021 / \mathrm{ct} 400420 \mathrm{r}$.

23. J. Contreras-García; R. A. Boto; F. Izquierdo-Ruiz; I. Reva; T. Woller; M. Alonso. A benchmark for the non-covalent interaction (NCI) index or... is it really all in the geometry? Theor. Chem. Acc., 135, 242 (2016). DOI: 10.1007/s00214-016-1977-7.

24. Q. Yang; Y. Liu; Z. Zhang; F. Zhou; Z. Wang; B. Zheng; T. Lu. Probing the halogen bond donation ability of multivalent At-center in $\mathrm{AtXn}(\mathrm{X}=\mathrm{Cl}, \mathrm{Br}, \mathrm{I} ; \mathrm{n}=1,3,5) \cdots \mathrm{H} 2 \mathrm{O} / \mathrm{H} 2 \mathrm{~S}$ complexes. Computational and Theoretical Chemistry, 1195, 113090 (2021). DOI: 10.1016/j.comptc.2020.113090.

25. F. Weigend; R. Ahlrichs. Balanced basis sets of split valence, triple zeta valence and quadruple zeta valence quality for H to Rn: Design and assessment of accuracy. Phys. Chem. Chem. Phys., 7, 3297-3305 (2005). DOI: 10.1039/b508541a.

26. J. F. Gonthier; S. N. Steinmann; L. Roch; A. Ruggi; N. Luisier; K. Severin; C. Corminboeuf. $\pi$ Depletion as a criterion to predict [small pi]-stacking ability. Chem. Commun., 48, 9239-9241 (2012). DOI: $10.1039 / \mathrm{c} 2 \mathrm{cc} 33886 \mathrm{f}$.

27. Z. Liu; T. Lu; Q. Chen. An sp-hybridized all-carboatomic ring, cyclo[18]carbon: Bonding character, electron delocalization, and aromaticity. Carbon, 165, 468-475 (2020). DOI: 10.1016/j.carbon.2020.04.099.

28. Z. Liu; T. Lu; S. Hua; Y. Yu. Aromaticity of Hückel and Möbius Topologies Involved in Conformation Conversion of Macrocyclic [32]Octaphyrin(1.0.1.0.1.0.1.0): Refined Evidence from Multiple Visual Criteria. J. Phys. Chem. C, 123, 18593-18599 (2019). DOI: 10.1021/acs.jpcc.9b06302.

29. T. Lu; Q. Chen. A simple method of identifying $\pi$ orbitals for non-planar systems and a protocol of studying $\pi$ electronic structure. Theor. Chem. Acc., 139, 25 (2020). DOI: 10.1007/s00214-019-2541-z.

30. Z. Liu; T. Lu; Q. Chen. An sp-hybridized all-carboatomic ring, cyclo[18]carbon: Electronic structure, electronic spectrum, and optical nonlinearity. Carbon, 165, 461-467 (2020). DOI: 10.1016/j.carbon.2020.05.023.

31. Z. Liu; T. Lu; Q. Chen. Intermolecular interaction characteristics of the all-carboatomic ring, cyclo[18]carbon: Focusing on molecular adsorption and stacking. Carbon, 171, 514-523 (2021). DOI: 10.1016/j.carbon.2020.09.048.

32. Z. Liu; T. Lu; Q. Chen. Vibrational spectra and molecular vibrational behaviors of all-carboatomic rings, cyclo[18]carbon and its analogues. Chem. - Asian J., 16, 56 (2021). DOI: 10.1002/asia.202001228. 33. T. Lu; Q. Chen. Ultrastrong regulation effect of electric field on all-carboatomic ring, cyclo[18]carbon. ChemPhysChem (2021). DOI: 10.1002/cphc.202000903.

34. J. Pipek; P. G. Mezey. A fast intrinsic localization procedure applicable for ab initio and semiempirical linear combination of atomic orbital wave functions. J. Chem. Phys., 90, 4916-4926 (1989). DOI: $10.1063 / 1.456588$. 
